J Burn Care Res. 2017 ; 38(1): e89-e94. doi:10.1097/BCR.0000000000000407.

\title{
The Impact of Illicit Drug Use On Outcomes Following Burn Injury
}

Erica I. Hodgman, MD, Madhu Subramanian, MD, Steven E. Wolf, MD, Brett D. Arnoldo, MD, Herb A. Phelan, MD, Michael W. Cripps, MD, and Kareem R. AbdelFattah, MD

Division of Burns, Trauma, and Critical Care, Department of General Surgery. University of Texas, Southwestern. Dallas, Texas

\section{Abstract}

Introduction-Illicit drug use is common among patients admitted following burn injury. We sought to evaluate whether drug abuse results in worse outcomes.

Methods-The National Burn Repository (NBR) was queried for data on all patients with drug testing results available. Outcomes included mortality, hospital length of stay (LOS), intensive care unit (ICU) LOS, and duration of ventilator support. Propensity score weighting was performed to control for age, alcohol use, burn size, gender, and etiology of burn.

Results-A total of 20,989 patients had drug screen data available; 11,642 (55.5\%) tested positive for at least one drug of abuse. Illicit drug use was associated with a higher proportion of patients with flame burn (53.2 vs 48.4\%) and larger average burn size (11.2 vs $9.5 \%$ total body surface area, $\mathrm{p}<0.001)$. Attempted suicide was more likely if the patient had used drugs ( $2.8 \mathrm{vs}$ $1.7 \%, \mathrm{p}<0.001)$. Drug use resulted in longer hospital and ICU LOS (14.2 vs 11.4 and 8.5 vs 5.6 days, $\mathrm{p}<0.001$ ), but did not increase the risk of mortality (5.7 vs 5.2, $\mathrm{p}=0.08$ ). After propensity score weighting, drug use did not impact mortality, hospital LOS, or duration of ventilator support, but did increase the average ICU LOS by 1.2 days $(\mathrm{p}=0.001)$.

Conclusions-Drug use does not affect mortality, hospital LOS, or duration of ventilator support among burned patients. After controlling for burn size, age, mechanism of injury, and gender, patients with a positive drug screen had an average increase in ICU LOS by one day.

\section{Keywords}

Illicit drugs; burns; outcomes; epidemiology

\section{Introduction}

Acute intoxication, due to alcohol or other substances, has been reported in nearly half of all burn injuries in the United States; whether the intoxication is plays a causative role is difficult to ascertain.[1,2] This finding is not merely of epidemiologic significance: several studies have reported worse outcomes, including mortality, hospital and intensive care unit

Corresponding Author: Kareem R. AbdelFattah, 5323 Harry Hines Boulevard E5.503c, Dallas, TX 75390-9158, Phone: 214-648-,

Fax: 214-648-8464, kareem.abdelfattah@utsouthwestern.edu.

All authors have no conflicts of interest to report. 
length of stay, and development of post-traumatic stress disorder among patients who were intoxicated at the time of injury.[2-4] Many patients with psychiatric and substance abuse comorbidities will remain in hospital longer due inability to care for their own wounds and/or lack of a reliable care giver.[3] Other studies have suggested that acute intoxication reduces both motor coordination and reaction times, resulting in a more extensive injury and/or an inhalation injury.[2] However, alcohol intoxication has also been shown to lead to increased intestinal bacterial overgrowth and translocation, leading to an increased risk sepsis and multi-system organ failure.[5, 6]

Most data that are currently available are from single institution studies, and recent data are limited. Our group sought to evaluate the impact of illicit drug use on outcomes on a decadelong national experience in the United States. We predicted that burn injuries sustained in association with illicit drug use would lead to increased risk of mortality and prolonged hospitalization.

\section{Methods}

The American Burn Association (ABA) maintains the National Burn Repository (NBR), which is a national database containing data from all ABA-verified and some non-verified centers across the United States and Canada. For this study, the NBR version 8.0 main and drug screen datasets were queried. Demographic and clinical data points collected included the following: age, gender, $\%$ total body surface area (TBSA) burned, mechanism of injury (scald, flame, contact, grease, electrical, other), presence of inhalational injury, and whether the injury was self-inflicted or a suicide attempt. The primary outcome was mortality; secondary outcomes included hospital and intensive care unit (ICU) length of stay (LOS), duration of ventilator support. In order to complete missing values and standardize mechanism of injury were possible, categorical variables were cross-referenced with free text fields from the treatment facility. The following substances were considered an illicit drug: benzodiazepines, opiates, marijuana, barbiturates, dissociative agents (e.g. lysergic acid diethylamide (LSD) or phencyclidine (PCP)), amphetamines, cocaine, or "other" (unspecified in the NBR); tricyclic antidepressants and sedative hypnotics were not considered illicit. Alcohol use, defined either a positive report by the treating facility or by a documentation of a non-zero blood alcohol level, was considered separately from drug use. When unique patients had conflicting drug screen results for the same encounter (e.g. one screen listed as negative and another positive for a specific drug), the negative result was excluded to ensure that we accounted for any and all possible drug use. Additionally, this database does not provide information regarding whether drug screens that are positive for opioids or benzodiazepines were iatrogenic; to be as conservative as possible we therefore chose to include all these patients in the drug abuse group. Patients admitted for non-burn injuries or with elective readmissions were excluded from analysis.

Demographics and clinical characteristics were compared between those with positive and negative drug screens using $\chi^{2}$, Fisher's exact test, two-tailed Student's t-tests, and one-way ANOVA as appropriate. In order to estimate a treatment effect for drug use that is unbiased by other covariates, we calculated a propensity score model of drug use based on patient age, gender, alcohol use, burn etiology, and \% TBSA. Propensity scoring was performed to 
control age, alcohol use, burn size, gender, and etiology of burn. First, propensity scores were calculated using multivariable logistic regression to predict whether a patient had a positive drug screen. The scores were then used as weights in a generalized linear model to calculate the average treatment effect on the treated (ATT); additional covariate adjustments were also performed to obtain a "doubly robust" estimate of the treatment effect. All analyses were calculated using R version 3.2.2 (R Development Core Team, Vienna, Austria); propensity scoring was completed using the "twang" and "survey" packages.

\section{Results}

Of the 172,640 entries in the main NBR dataset, 21,570 patients (12.5\%) had drug screen results available; 581 of these were admitted electively or for management of a non-burn condition and were therefore excluded, yielding a sample size of 20,989 (Figure 1). Drug use was common in this sample, with a drug screen positive for any illicit drug reported in $11,642(55.5 \%)$ patients. The most common drug of abuse was opiates $(n=7,050,33.6 \%)$, followed by stimulants (including cocaine and amphetamines ( $\mathrm{n}=3,430,16.3 \%)$, marijuana $(\mathrm{n}=3,222,15.4 \%)$, and "other" drugs $(\mathrm{n}=2,660,12.7 \%)$. Most patients tested positive for only one drug; polysubstance abuse was identified in a small minority $(n=68.0 .3 \%)$.

Selected demographics of drug-users and non-drug users are presented in Table 1. On average, patients who tested positive for illicit drug use tended to be slightly older (38.3 vs 35.4 years old, $\mathrm{p}<0.001)$ and have a larger injury (9.5 vs $11.2 \%$ TBSA, $\mathrm{p}<0.001)$. There was a small but significant increase in the rate of inhalation injury among intoxicated patients as well $(10.7 \mathrm{vs} 8.7 \%, \mathrm{p}<0.001)$. Although men were more likely to sustain a burn in general, there was a relatively larger proportion of men among patients with a positive drug screen at the time of admission (75.9 vs $73.2 \%, \mathrm{p}<0.001)$. Self-inflicted injuries/ suicide attempts were relatively uncommon, occurring in only 489 or $2.3 \%$ of the population, but drug abuse played a factor in two-thirds of these attempts $(\mathrm{n}=331,67.7 \%, \mathrm{p}<0.001)$. Although unadjusted mortality was not increased among illicit drug users (5.7 vs $5.2 \%, \mathrm{p}=0.08$ ), hospital LOS (14.2 vs 11.4 days, $\mathrm{p}<0.001)$ and ICU LOS ( 8.5 vs 5.6 days, $\mathrm{p}<0.001$ ), and duration of ventilator support (5.3 vs 3.5 days, $\mathrm{p}<0.001$ ) were all significantly longer. Patients with a positive drug screen were also significantly less likely to be able to return home without home health services (67.5 vs $74.5 \%$, p<0.001).

When comparing outcomes based on different patterns of drug use, patients who tested positive for marijuana alone $(\mathrm{n}=972)$ tended to more closely resemble those who used no drugs ( $\mathrm{n}=9347$ ) compared with patients who used other drugs alone $(\mathrm{n}=8420)$ or marijuana together with other drugs $(n=2250)$ (Table 2). Marijuana users tended to be younger, with a mean age of 32.3 years, versus non-users (mean 35.4 years) or those who used other drugs (mean 40.3 years). Mean burn size was smaller (9.5\% for non-users, and 9.2 for marijuana users) compared with those who used other drugs (11.5\% for users of other drugs, and $11.9 \%$ for users of marijuana and other drugs). While flame burns remain most common among all four groups, scald burns were found in over $20 \%$ of non-users, vs $8.5-11.1 \%$ of drug users. Patients who used other drugs, with or without marijuana, tended to have significantly longer hospital LOS, ICU LOS, and duration of ventilator support than their counterparts who used no drugs or marijuana alone. Interestingly, the mortality rates 
between non-users and marijuana users are similar (5.2 and 5.3\% respectively), while the mortality rate among users of drug users was increased (6.5\%) and for marijuana plus other drug users was actually the lowest $(3.2 \%, \mathrm{p}<0.001)$.

To evaluate the contribution of alcohol use to these observed outcomes, we also compared patients who used drugs alone ( $\mathrm{n}=10972)$, those who used both drugs and alcohol $(\mathrm{n}=670)$, those who had only positive alcohol screens $(\mathrm{n}=229)$, and those whose screens were negative for both alcohol and drugs ( $\mathrm{n}=9118$ ) (Table 3). In general, patients who did not drink or use any illicit drugs had a decreased hospital LOS, ICU LOS, and duration of ventilator support. Compared with all other groups, patients who ingested alcohol alone had the highest risk of death $(10.5 \%, \mathrm{p}=0.001)$. Patients who were not under the influence of alcohol or drugs were significantly less likely to be burned as a result of a suicide attempt $(1.7 \%, \mathrm{p}<0.001)$.

To perform the propensity scoring analysis, an additional 7,325 patients were excluded due to missing covariate data; therefore the scoring was performed on a final sample size of 13,664 (Figure 1). After weighting to adjust for the effect of age, sex, \%TBSA burned, mechanism of injury, and alcohol use, illicit drug use did not result in a statistically significant increase in the risk of mortality (OR 1.00, $\mathrm{p}=0.46)$, hospital length of stay ( 0.38 days, $\mathrm{p}=0.52$ ), or duration of ventilator support ( 0.82 days, $\mathrm{p}=0.18)$. However, among those who used drugs, ICU length of stay was increased by 1.23 days $(\mathrm{p}=0.0013)$. Additional covariate adjustment with the same variables used in the propensity scoring did not alter the risk of mortality, hospital LOS, or number of ventilator days; ICU LOS remained significantly longer (1.08 days, $\mathrm{p}=0.0009$ ).

\section{Discussion}

Data on the effect of drug use on outcomes following burn injury are limited in the currently available literature. Due in large part to the fact that alcohol consumption is more common than illicit drug use, prior studies have focused more on the role of alcohol as both a predisposing factor for injury and as a predictor of outcomes.[7] Traditionally, alcohol has been thought to have three effects on the risk of both the injury itself as well as subsequent complications or death.[7, 8] First, inebriated patients tend to be more reckless and therefore have a higher risk of injury; per Soderstrom et al, "alcohol and logic do not mix".[8] Secondly, intoxicated patients have diminished coordination and/ or altered mental status, causing them to have more difficulty in escaping the source of injury and resulting in deeper and/or larger wounds and a higher risk of inhalation injury.[9] Lastly, it is also thought that chronic alcohol use results in physiologic changes that contribute independently to an increased risk of complications among those who initially survive their initial resuscitation. $[6,10]$

Based on our findings, we believe that, like alcohol consumption, illicit drug use influences the risk of burn injury as well as injury size and severity. As in the other studies on drug and alcohol use among burn patients, we found that intoxicated patients were more likely to have a flame burn than their sober counterparts.[3, 7, 11, 12] Moreover, patients who used illicit drugs of any sort had a significantly larger burden of injury, and were also more likely to sustain an inhalation injury than those who did not use drugs. In particular, the effect on burn 
size seems to be due more to drugs other than marijuana, as average burn size among those who used marijuana only was quite similar to that of nondrug users at around 9-9.5\% TBSA, while those who used other drugs (with or without marijuana) had burn sizes of 11.5-11.9\% TBSA. This finding contrasts slightly with the findings of Jehle et al, who performed a similar analysis of the NBR and found that marijuana use resulted in an increased hospital LOS; however, their study scope was limited to use of cannabis alone, and did consider whether patients had used other illicit substances.[12]

As in other studies, we found that drug use appears to influence not only the risk of injury severity, but also hospital and intensive care unit LOS, and by extension, resource utilization. [3] On unadjusted analysis, patients who abused any illicit drugs had longer hospital and intensive care unit stays, but not a higher risk of mortality. After stratifying based on the pattern of drug use, marijuana alone does not appear to worsen outcomes, while use of other drugs seem to result in the longest hospitalization and highest risk of death. Interestingly, the use of marijuana in addition to these other drugs appears to temper this increased risk. Most compellingly, the effect of drugs of abuse on ICU stay persisted even after using propensity scoring to control for alcohol use as well as the other classic drivers of morbidity and mortality among burn patients (\%TBSA, age, mechanism). Jehle et al did find a significantly increased length of stay among cannabis users, however based on our data it appears that use of other drugs may play a confounding role in this result.[12]

Interestingly, the highest mortality rate $(10.5 \%)$ in this study was seen among the small subset of patients who drank alcohol but did not use any illicit substances, including cannabis. Haum et al found a non-linear relationship between blood alcohol content and risk of mortality; they postulated that the non-linearity stemmed from the fact that alcoholics tended to have higher blood alcohol levels and that their tolerance would have rendered them less impaired than their counterparts with lower alcohol levels.[2]

In our sample, roughly two-thirds of all patients who were reported to have a self-inflicted injury had a drug screen result positive for illicit drugs. Unfortunately, with this sample it is not possible to tease out whether both drug abuse and suicidality are signs of comorbid psychiatric illness, or if the intoxication reduces the inhibitions of a patient who would otherwise be able to resist the impulse to inflict self-harm. A national survey of admissions to Emergency Department following a self-inflicted injury or suicide attempt found that over half of all patients were coded as having a mental illness, and $9 \%$ of patients were coded as having an alcohol abuse disorder.[13] This reinforces the presence of a significant amount of overlap among the populations of patients who have a psychiatric disorder, who abuse alcohol and/or illicit substances, and who intentionally harm themselves. Unfortunately, preexisting psychiatric illness may also make it more difficult for patients to adjust following their injuries and to handle the sequelae (including painful, prolonged wound care regimens and the diminished ability to function independently).[3] Moreover a prolonged hospital ICU length of stay may actually result in an increased risk of post traumatic stress disorder among those patients with a history of pre-existing mental illness or prior unemployment.[4] We believe that these more vulnerable patients may benefit from more aggressive interventions by a multidisciplinary team, including social workers and mental health professionals, to both delineate the nature and extent of pre-existing mental health 
conditions, as well as to mitigate the potential negative psychological consequences of a burn injury.

The greatest limitation of this study is the risk of significant bias that stems from the lack of a standardized approach to drug screening among facilities. As reported in a review of drug and alcohol testing in a general trauma population, routine universal screening is typically not performed in any center, and there is likely significant selection bias in deciding whom to screen.[14] Given that only roughly 20,000 patients had drug testing data available in a database of over 172,000 admissions, it seems likely that we are missing many patients for whom illicit drug use plays a role, either directly or indirectly, in their injury. We propose that a more standardized approach to drug and alcohol screening may identify additional atrisk patients who would benefit from added resource availability and intensive therapy.

\section{Acknowledgments}

External funding: Support provided by Sons of the Flag and the Pettis Family

\section{References}

1. McGill V, Kowal-Vern A, Fisher SG, Kahn S, Gamelli RL. The Impact of Substance Use on Mortality and Morbidity from Thermal Injury. The Journal of trauma. 1995:38. [PubMed: 7745655]

2. Haum A, Perbix W, Hack HJ, Stark GB, Spilker G, Doehn M. Alcohol and drug abuse in burn injuries. Burns : journal of the International Society for Burn Injuries. 1995; 21:194-9. [PubMed: 7794500]

3. Alexander W, Coghlan P, Greenwood JE. A 365-day view of the difficult patients treated in an Australian adult burn center. Journal of burn care \& research : official publication of the American Burn Association. 2015; 36:e146-52. [PubMed: 25522153]

4. Davydow DS, Katon WJ, Zatzick DF. Psychiatric morbidity and functional impairments in survivors of burns, traumatic injuries, and ICU stays for other critical illnesses: a review of the literature. Int Rev Psychiatry. 2009; 21:531-8. [PubMed: 19919206]

5. Choudhry MA, Rana SN, Kavanaugh MJ, Kovacs EJ, Gamelli RL, Sayeed MM. Impaired intestinal immunity and barrier function: a cause for enhanced bacterial translocation in alcohol intoxication and burn injury. Alcohol. 2004; 33:199-208. [PubMed: 15596088]

6. Kavanaugh MJ, Clark C, Goto M, Kovacs EJ, Gamelli RL, Sayeed MM, et al. Effect of acute alcohol ingestion prior to burn injury on intestinal bacterial growth and barrier function. Burns : journal of the International Society for Burn Injuries. 2005; 31:290-6. [PubMed: 15774282]

7. Barillo DJ, Goode R. Substance abuse in victims of fire. The Journal of burn care \& rehabilitation. 1996; 17:71-6. [PubMed: 8808362]

8. Soderstrom CA, Trifillis AL, Shankar BS. Marijuana and alcohol use among 1023 trauma patients. Archives of surgery. 1987; 123:733-7.

9. Davis CS, Esposito TJ, Palladino-Davis AG, Rychlik K, Schermer CR, Gamelli RL, et al. Implications of alcohol intoxication at the time of burn and smoke inhalation injury: an epidemiologic and clinical analysis. Journal of burn care \& research : official publication of the American Burn Association. 2013; 34:120-6. [PubMed: 23079566]

10. Kelley D, Lynch JB. Burns in alcohol and drug users result in longer treatment times with more complications. The Journal of burn care \& rehabilitation. 1992; 13:218-20. [PubMed: 1587921]

11. Holmes WJ, Hold P, James MI. The increasing trend in alcohol-related burns: it's impact on a tertiary burn centre. Burns : journal of the International Society for Burn Injuries. 2010; 36:93843. [PubMed: 20185245]

12. Jehle CC Jr, Nazir N, Bhavsar D. The rapidly increasing trend of cannabis use in burn injury. Journal of burn care \& research : official publication of the American Burn Association. 2015; 36:e12-7. [PubMed: 25412052] 
13. Doshi A, Boudreaux ED, Wang N, Pelletier AJ, Camargo CA Jr. National study of US emergency department visits for attempted suicide and self-inflicted injury, 1997-2001. Annals of emergency medicine. 2005; 46:369-75. [PubMed: 16183394]

14. Dunham CM, Chirichella TJ. Trauma activation patients: evidence for routine alcohol and illicit drug screening. PloS one. 2012; 7:e47999. [PubMed: 23094103] 


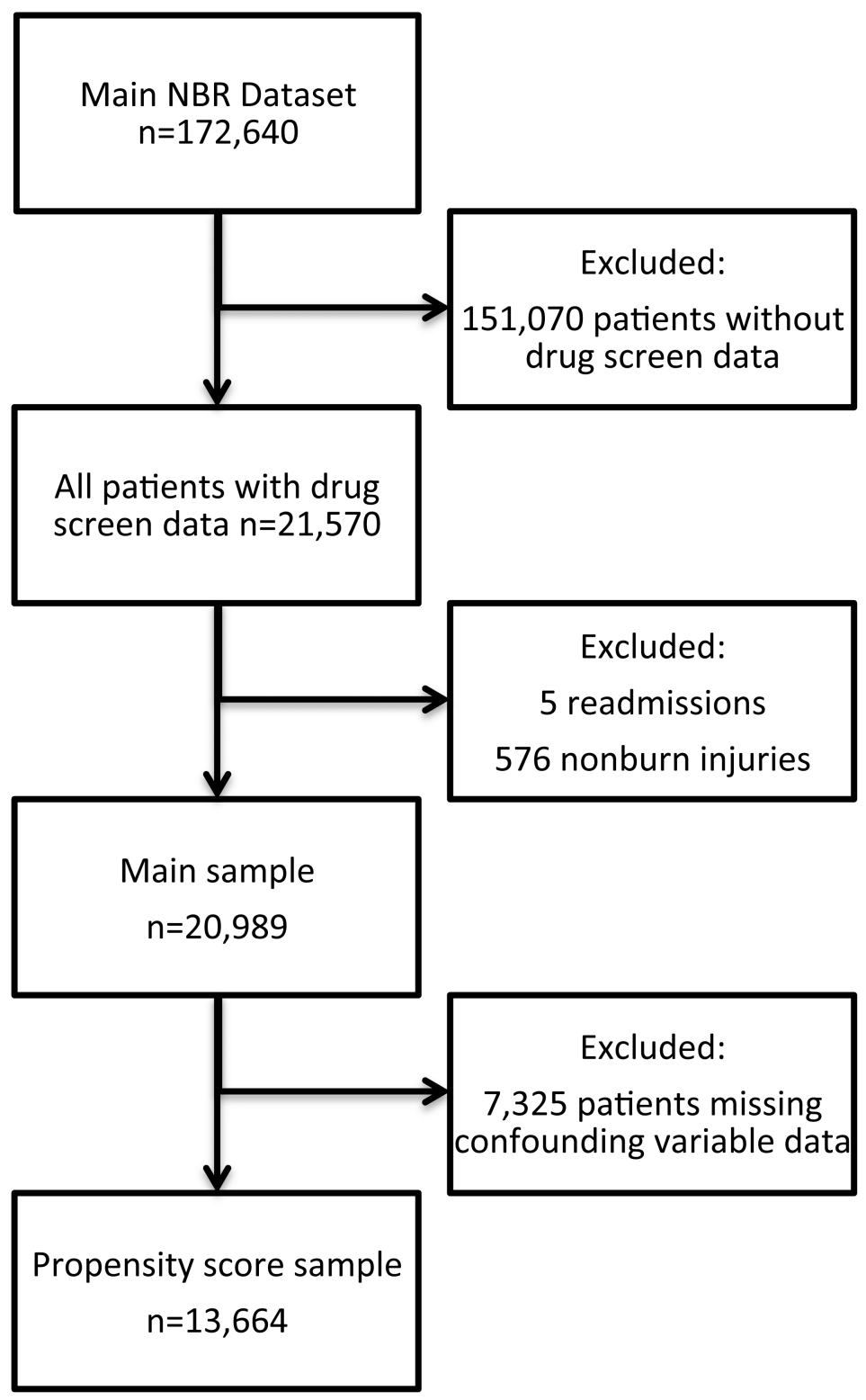

Figure 1. Consort Diagram 
Table 1

Selected Demographics of Drug Users and Non-Drug Users

\begin{tabular}{|l|l|l|l|}
\hline & Non-Drug Users & Drug Users & $\mathbf{p}$ \\
\hline Age (Median [IQR]) & $35.4[19.2-51.3]$ & $38.3[25.4-49.4]$ & $<0.001$ \\
\hline Gender (\% male) & $2370(47.0 \%)$ & $2670(52.1 \%)$ & $<0.001$ \\
\hline$\%$ TBSA (Median, [IQR]) & $9.5[1.5-11.0]$ & $11.2[1.0-14.5]$ & $<0.001$ \\
\hline Mechanism & & & $<0.001$ \\
\hline Flame & $4528(48.4 \%)$ & $6195(53.2 \%)$ & \\
\hline Scald & $1961(21.0 \%)$ & $1245(10.7 \%)$ & \\
\hline Contact & $687(7.4 \%)$ & $330(2.8 \%)$ & \\
\hline Electrical & $388(4.1 \%)$ & $486(4.2 \%)$ & \\
\hline Chemical & $282(3.0 \%)$ & $315(2.7 \%)$ & \\
\hline Grease & $259(2.8 \%)$ & $315(2.7 \%)$ & \\
\hline Other & $1242(13.3 \%)$ & $2756(23.7 \%)$ & \\
\hline Inhalation Injury & $773(8.7 \%)$ & $1186(10.7 \%)$ & $<0.001$ \\
\hline Suicide/ Self-Inflicted & $158(1.7 \%)$ & $331(2.8 \%)$ & $<0.001$ \\
\hline Injury due to Assault or Abuse & $194(2.1 \%)$ & $273(2.4 \%)$ & 0.19 \\
\hline Hospital Disposition on Discharge & & & $<0.001$ \\
\hline Home, without home health & $6959(74.5 \%)$ & $7858(67.5 \%)$ & \\
\hline Home, with home health & $342(3.7 \%)$ & $688(5.9 \%)$ & \\
\hline Rehab & $374(4.0 \%)$ & $753(6.5 \%)$ & \\
\hline Skilled Nursing Facility & $316(3.4 \%)$ & $373(3.2 \%)$ & \\
\hline Jail or Law Enforcement & $29(0.3 \%)$ & $101(0.9 \%)$ & \\
\hline Mortality & $457(5.2 \%)$ & $635(5.7 \%)$ & 0.078 \\
\hline Hospital LOS & $4.0[1.0-13.0]$ & $7.0[2.0-17.0]$ & $<0.001$ \\
\hline ICU LOS & $0.0[0.0-3.0]$ & $1.0[0.0-8.0]$ & $<0.001$ \\
\hline Ventilator Days & $0.0[0.0-0.0]$ & $0.0[0.0-1.0]$ & $<0.001$ \\
\hline & & & \\
\hline & & & \\
\hline & & & \\
\hline & & & \\
\hline & & & \\
\hline & & & \\
\hline & & & \\
\hline
\end{tabular}




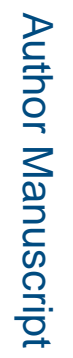

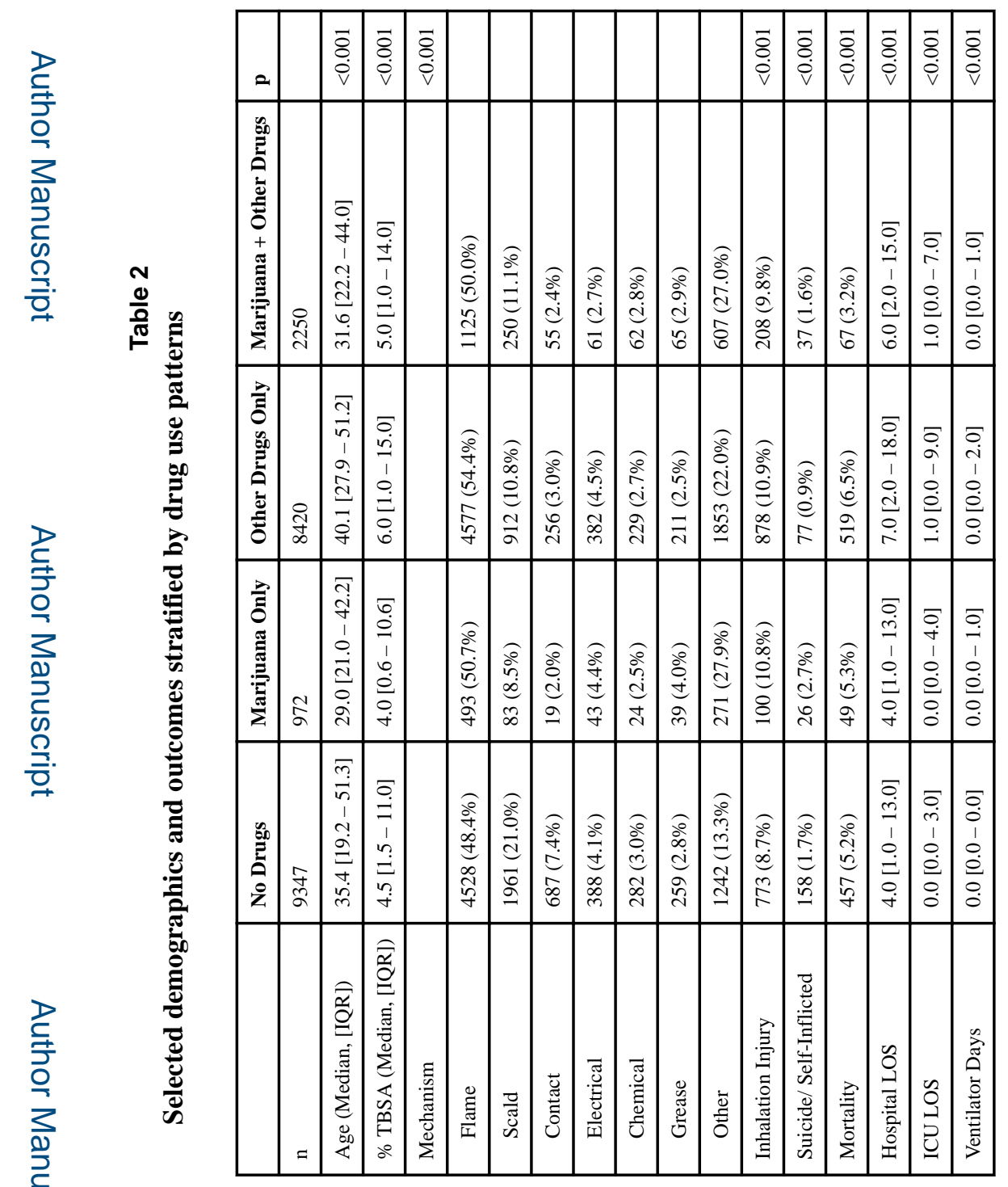

J Burn Care Res. Author manuscript; available in PMC 2018 January 01. 


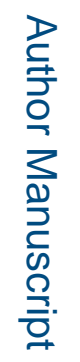

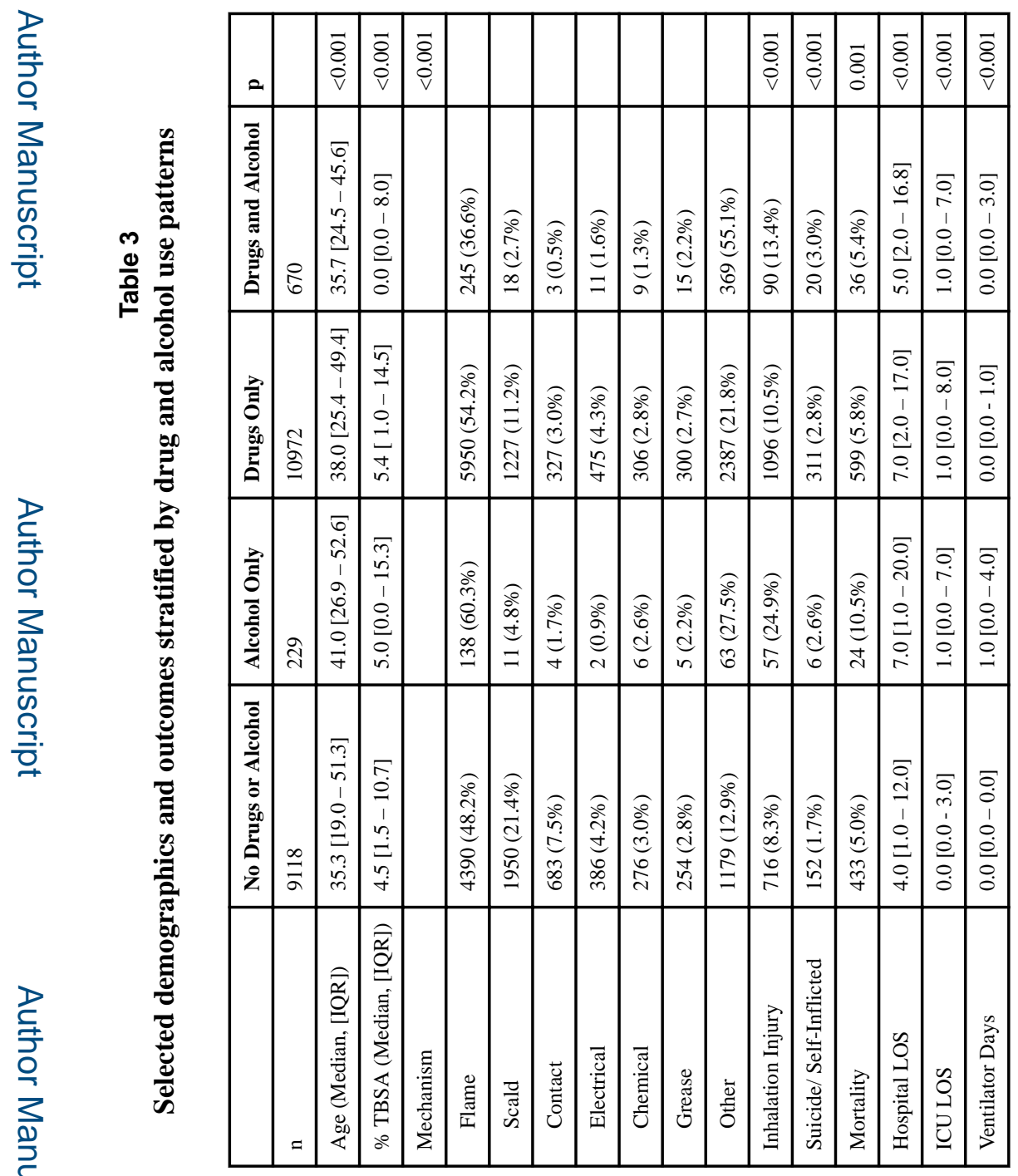

J Burn Care Res. Author manuscript; available in PMC 2018 January 01. 